\title{
On the Latency of IEEE 802.11ax WLANs with Parameterized Spatial Reuse
}

\author{
Eloise de Carvalho Rodrigues ${ }^{\star \dagger}$, Adrian Garcia-Rodriguez ${ }^{\star}$, Lorenzo Galati Giordano ${ }^{\star}$, and Giovanni Geraci ${ }^{\dagger}$ \\ ${ }^{\star}$ Nokia Bell Labs, Dublin, Ireland \\ ${ }^{\dagger}$ Universitat Pompeu Fabra (UPF), Barcelona, Spain
}

\begin{abstract}
In this article, we evaluate the performance of the parameterized spatial reuse (PSR) framework of IEEE 802.11ax, mainly focusing on its impact on transmission latency. Based on detailed standard-compliant system-level simulations, we provide a realistic analysis of the effects of PSR considering different scenario densities, traffic loads, and access points (APs) antenna capabilities to quantify its performance gains under various scenarios. Our results show that, in medium-density scenarios, PSR can offer up to a $3.8 \times$ reduction in the $5 \%$ worst-case latencies for delay-sensitive stations with respect to an 802.11ax system without PSR. Moreover, our study demonstrates that, for low-latency communications, providing the network with PSR capabilities may be an appealing alternative to the deployment of more costly multi-antenna APs.
\end{abstract}

\section{INTRODUCTION}

From our houses to smart cities and factories, Wi-Fi products are widely used due to the simplicity of the IEEE 802.11 protocols combined with low-cost deployment and management. From 2020 to 2023, the number of Wi-Fi 6 hotspots-based on IEEE 802.11ax-is foreseen to increase 13 fold [1]. As the number of concurrently connected devices increases, one of the main challenges to overcome is the potential delay introduced by the mandatory listen-before-talk (LBT) adopted by technologies using the unlicensed spectrum [2]. LBT imposes that a device willing to access the channel must listen to the medium and guarantee that there are no other ongoing transmissions before attempting to transmit. This mechanism is a potential show stopper for low-latency applications, e.g., Industry 4.0 and autonomous robots.

A way to address this problem is by increasing spatial reuse (SR). Two related techniques are included in the IEEE 802.11ax amendment: overlapping basic service set packet detect (OBSS/PD) and parameterized spatial reuse (PSR) [3]. OBSS/PD consists of relaxing the clear channel assessment (CCA) signal detection threshold to increase the probability of concurrent transmissions. Instead, PSR takes advantage of the trigger frame (TF) that precedes uplink (UL) transmissions to permit access points (APs) to control spatial reuse. According

This work was supported by the European Union's Horizon 2020 research and innovation programme under Marie Skłodowska-Curie Grant agreement No.812991 (PAINLESS). The work of G. Geraci was supported in part by MINECO under Project RTI2018-101040-A-I00 and by the Postdoctoral Junior Leader Fellowship Programme from "la Caixa" Banking Foundation.

${ }^{1}$ Referred to as spatial reuse parameter (SRP) until Standard Draft 5.0. to the information conveyed in the TF, non-associated stations (STAs) determine whether they are allowed to access the channel based on the potential interference they may generate towards the AP that transmitted the TF. This mode of operation can be advantageous for critical applications with low-latency requirements. Indeed, IEEE $802.11 \mathrm{be}$ - the basis of the nextgeneration Wi-Fi standard-is currently discussing how to further reduce worst-case latencies by building on top of the PSR framework [4]-[7]. In these circumstances, assessing the gains already provided by the PSR scheme available in IEEE 802.11ax is of crucial importance.

Related works surveying the main functionalities and operation of both OBSS/PD and PSR spatial reuse include [8][12], while simulation-based performance evaluations of the OBSS/PD SR technique are provided in [11], [13], [14]. To the best of our knowledge, there are currently no works on the literature characterizing PSR's performance. This is mainly due to the complexity of implementing and evaluating the PSR functionality since an accurate analysis also requires the implementation of intricate 802.11ax features such as TF-based operations and UL multi-user multiple-input and multiple-output (MU-MIMO) [11].

In this paper, we compare the performance of an IEEE 802.11ax system with and without PSR capabilities. In particular, we analyze how PSR affects latency in practical scenarios with various deployment densities, traffic loads, and number of AP antennas. To do so, we implement the PSR framework as specified by IEEE 802.11ax, accounting for its complex features and the latest 3GPP 3D channel model, all leading to accurate results. Our main takeaways can be summarized as follows:

- For high-, medium-, and low-density deployments with high traffic loads, PSR can reduce the 5\% worst-case latencies with respect to the baseline IEEE 802.11 ax operations by a factor $1.8,3.8$, and 3.4, respectively.

- Allowing a more aggressive spatial reuse through PSR provides more remarkable latency benefits under higher traffic loads - where the wireless medium remains generally occupied-than under low-to-medium loads.

- Latency-wise, a PSR system with single-antenna APs can outperform eight-antenna APs without spatial reuse capabilities, designating PSR as an alternative to more costly multi-antenna APs. 


\section{PSR-BASED SPATIAL REUSE OPERATION}

In this section, we describe both baseline (Sec. II-A) and PSR-enabled (Sec. II-B) IEEE 802.11ax operations, providing examples to highlight the relevance of PSR for low-latency applications.

\section{A. Baseline 802.11ax Operation}

In the baseline 802.11ax operation, the transmission is done through carrier sense multiple access/collision avoidance (CSMA/CA). This entails that APs and STAs need to perform i) physical and ii) virtual carrier sensing before initiating a transmission. Physical carrier sensing determines whether the channel is occupied or not by listening to wireless signal energy. Virtual carrier sense relies on the transmitter informing the surrounding nodes about the length of the imminent data transmissions. The STAs receiving this signaling set their network allocation vector (NAV) that indicates for how long they must defer from accessing the medium. The medium is sensed as "busy" if the STA/AP either i) senses signals at or above $-62 \mathrm{dBm}$, and/or ii) the NAV is set [15]. Once an AP gains channel access in TF-based operations, it transmits a TF to initiate an uplink transmission of one or multiple STAs. The TF is a control frame containing information about the scheduled stations and other relevant information.

Fig. 1 (a) illustrates legacy 802.11 operations. In the example, we have two basic service sets (BSSs): BSS 1, composed by $\mathrm{AP}_{1}$ and $\mathrm{STA}_{11}$, and $\mathrm{BSS} 2$, composed by $\mathrm{AP}_{2}, \mathrm{STA}_{21}$ and $\mathrm{STA}_{22}$. The STAs from BSS 2 are latency-sensitive stations, meaning that their packets are short and must be delivered in a timely manner. In this example, $\mathrm{AP}_{1}$ is going to schedule a long uplink transmission from $\mathrm{STA}_{11}$ (e.g., in the order of several milliseconds). In Fig. 1(a), once $\mathrm{AP}_{1}$ gains channel access in accordance with the aforementioned rules, the operation continues as follows:

1) $\mathrm{AP}_{1}$ triggers an uplink transmission from $\mathrm{STA}_{11}$;

2) $\mathrm{STA}_{21}$ and $\mathrm{STA}_{22}$ set their NAV indicating that the channel is busy and defer from channel access until the NAV expires;

3) $\mathrm{STA}_{21}$ and $\mathrm{STA}_{22}$ contend for channel access again. $\mathrm{STA}_{21}$ gets channel access first, and $\mathrm{STA}_{22}$ will defer from channel access.

In this example, the latency-sensitive STAs $\left(\mathrm{STA}_{21}\right.$ and $\left.\mathrm{STA}_{22}\right)$ may suffer for having to wait for the long transmission of $\mathrm{STA}_{11}$ to be finished before accessing the channel and transmitting their short packets, which have a negative impact on their perceived quality of service.

\section{B. PSR Operation}

The PSR-based spatial reuse mechanism was included in the IEEE 802.11ax standard to allow the wireless medium to be reused more often in dense deployment scenarios [3], [8]. Compared to other SR techniques such as OBSS/PD, PSR facilitates a more controlled spatial reuse. This control is achieved because APs, the transmission holders or donors,

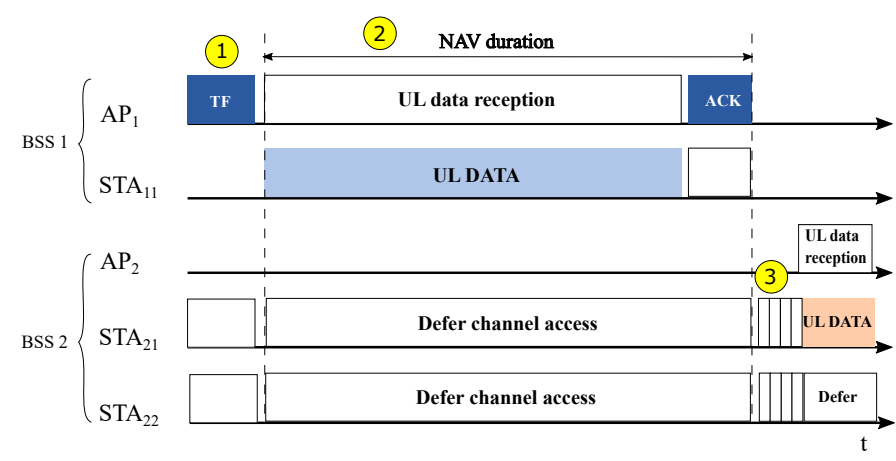

(a) Baseline 802.11ax scenario

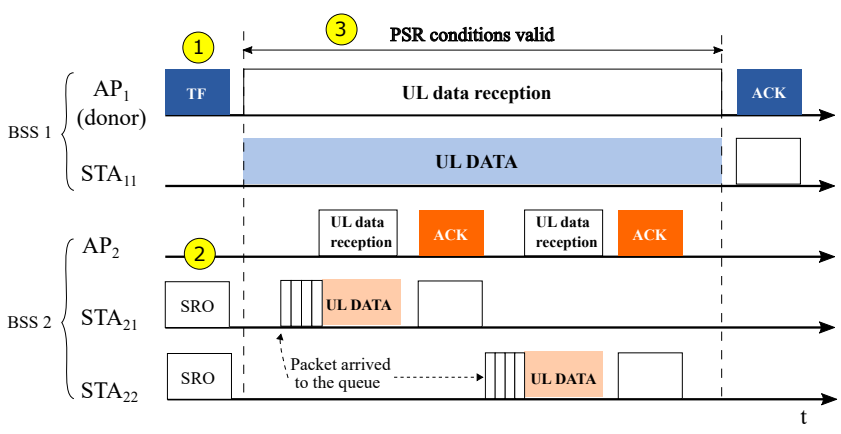

(b) PSR-enabled scenario

Fig. 1. Illustration of (a) baseline operation for an IEEE 802.11ax system using CSMA/CA, and (b) a PSR-capable IEEE 802.11ax system.

only provide to non-associated STAs the possibility of accessing the channel during the uplink-triggered transmissions as long as they comply with a specified acceptable level of interference, devised so as not to harm the uplink of own served STAs. In other words, the donor APs that want to facilitate spatial reuse do so in such a way that the performance of its scheduled uplink transmissions is guaranteed. For this, the AP defines an acceptable interference level, $\mathrm{I}_{\mathrm{AP}}$, as the maximum interference that can be perceived by the transmission holder without compromising its transmission. This acceptable interference is a value in $\mathrm{dBm}$ calculated as:

$\mathrm{I}_{\mathrm{AP}}=\mathrm{UL} \_$Target_RSSI - Min_SNR_MCS - Safety_Margin,

where UL_Target_RSSI is the UL target receive signal strength indicator (RSSI) in dBm indicated in the TF, Min_SNR_MCS is the minimum signal to noise ratio (SNR) value that yields $\leq 10 \%$ packet error rate (PER) for the highest modulation and coding scheme (MCS) of the following uplink data transmission, and a Safety_Margin that should not exceed $5 \mathrm{~dB}$.

To make sure that the interference received is below $\mathrm{I}_{\mathrm{AP}}$, the AP includes the PSR_INPUT field in the TF. The value of PSR_INPUT is computed as:

$$
\text { PSR_INPUT }=\text { TX_PWR } A P+I_{A P}
$$

where TX_PWR $\mathrm{AP}$ is the power used by the AP to transmit the trigger frame in $\mathrm{dBm}$.

A STA with PSR capabilities that receives a TF allowing PSR is allowed to resume the channel access contention 
procedure and initiate a transmission after identifying what is called a spatial reuse opportunity (SRO) if the following conditions, designed to avoid interference in reception for the AP that holds the current transmission, are met:

Condition 1: The TF received by the STA is an inter-BSS trigger frame, i.e. a trigger frame sent from an AP located in a BSS different from the BSS in which the STA willing to use SR is associated;

Condition 2: The intended transmission power TX_PWR should not exceed the interference limit set by the AP granting the spatial reuse opportunity. This condition is formally defined as:

TX_PWR STA $-10 \log _{10}\left(\frac{\text { TX_BW }}{20 \mathrm{MHA}}\right) \leq$ PSR_INPUT - RPL,

where TX_BW STA $_{\text {ST }}$ is the intended transmission bandwidth in $\mathrm{MHz}$ and RPL is the received power level that indicates the receiving power of TF [8].

Importantly, the conditions set by the AP allowing PSR are only valid for the duration of the uplink data transmission that follows the TF. This entails that the STA intending to transmit during an SR opportunity should make sure that its transmission duration is smaller than the uplink-triggered transmission initiated by the donor AP. Once the STA decides to take the PSR opportunity, it may ignore the network allocation vector (NAV), but it must still perform physical carrier sensing.

To summarize the process, consider the example in Fig. 1 (b) in which the APs and the latency-sensitive STAs now have spatial reuse capabilities. The PSR operation, in this example, would proceed as follows:

1) The donor $\mathrm{AP}_{1}$ triggers $\mathrm{STA}_{11}$ for uplink transmission and sets the trigger frame field that allows PSR, also setting PSR_INPUT;

2) $\mathrm{STA}_{21}$ and $\mathrm{STA}_{22}$ receive an inter-BSS trigger frame allowing PSR. Both stations can grab spatial reuse opportunities as we assume that Conditions 1 and 2 are met;

3) As the transmission triggered by $\mathrm{AP}_{1}$ is very long, both $\mathrm{STA}_{21}$ and $\mathrm{STA}_{22}$ are able to transmit their short packets within the currently set spatial reuse opportunity as soon as the packets arrive in the queue.

This example clearly illustrates how PSR may help to reduce the worst-case latencies of the STAs with latency constraints by taking advantage of the multiple short-packet transmissions that may be performed within a long uplinktriggered transmission.

\section{PSR PERformance Evaluation}

In this section, we consider the scenario deployment described in Sec. ЩII-A to extensively evaluate the impact of deployment densities (Sec. III-B), traffic loads (Sec. 【II-C), and the number of antennas at the APs (Sec. III-D) on the performance of two 802.11ax systems: a baseline one without PSR capabilities, and an upgraded one where devices have PSR capabilities. To evaluate the performance of each scenario, we run standard-compliant system-level simulations

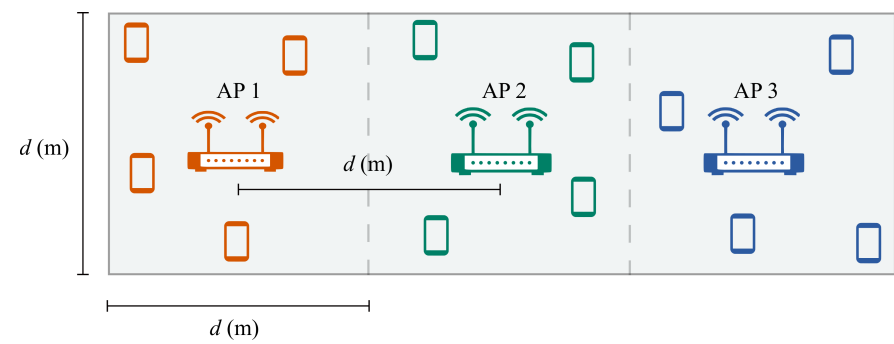

Fig. 2. Illustration of a $3 d \times d$ indoor scenario with three ceiling-mounted Wi-Fi APs separated by a distance $d$ and $s$ STAs uniformly distributed.

TABLE I

SYSTEM PARAMETERS

\begin{tabular}{|l|l|}
\hline Parameter & Description \\
\hline AP deployment & 3 ceiling mounted APs; AP height $=3 \mathrm{~m}$ \\
\hline STA deployment & $\begin{array}{l}16 \text { broadband STAs, 8 low-latency STAs; 2D } \\
\text { uniform distribution; STA height }=1 \mathrm{~m}\end{array}$ \\
\hline STA-AP association & Strongest average received signal \\
\hline Frequency/Bandwidth & $5.18 \mathrm{GHz} / 80 \mathrm{MHz}(1 \mathrm{channel})$ \\
\hline AP/STA max. TX power & $24 \mathrm{dBm} / 15 \mathrm{dBm}$ \\
\hline AP/STA noise figure & $7 \mathrm{~dB} / 9 \mathrm{~dB}$ \\
\hline Num. antennas per STA & 1 omni. antenna \\
\hline AP spatial Filter & Zero Forcing \\
\hline AP/STA sensitivity & $-90 \mathrm{dBm}$ \\
\hline PSR Safety_Margin & $3 \mathrm{~dB}$ \\
\hline MCS selection & SINR-driven \\
\hline STA scheduling & Round-Robin scheduling policy \\
\hline Max. TXOP length & $4 \mathrm{~ms}$ \\
\hline Broadband traffic & FTP3 [16] with a file size of 0.5 MBytes \\
\hline Low-latency traffic & $\begin{array}{l}\text { Augmented reality [17] with file size of 32 } \\
\text { bytes and constant file arrival of } 10 \mathrm{~ms}\end{array}$ \\
\hline Channel model & $\begin{array}{l}\text { 3D spatial channel model (3GPP TR38.901 } \\
- \text { InH [18]) }\end{array}$ \\
\hline AP/STA MAC conf. & $\begin{array}{l}\text { IP/MAC header overhead considered; No } \\
\text { EDCA; No RTS/CTS }\end{array}$ \\
\hline AP/STA PHY conf. & $\begin{array}{l}\text { PHY header overhead considered; 11ax } \\
\text { OFDM numerology with cyclic prefix dura- } \\
\text { tion }=0.8 \mu \text { s; Perfect channel state informa- } \\
\text { tion acquisition }\end{array}$ \\
\hline Simulations setup & $\begin{array}{l}\text { Monte Carlo simulations, 10 drops of 2 s for } \\
\text { each scenario }\end{array}$ \\
\hline
\end{tabular}

that account for regulatory constraints, accurate channel and traffic modeling and the main IEEE 802.11ax features.

\section{A. System Configuration}

The deployed scenario, illustrated in Fig. 2, consists in a $3 d \times d$ single-floor indoor wireless local area network (WLAN). This scenario comprises three ceiling-mounted WiFi APs at the height of $3 \mathrm{~m}$ separated by a distance $d$ and 24 STAs uniformly deployed at the height of $1 \mathrm{~m}$ in the considered area. The stations associate to the APs based on the strongest average received signal strength (RSS). We assume that, initially, all the APs are equipped with four antennas, and all the STAs are equipped with one omnidirectional antenna. As PSR operates in uplink, only uplink transmissions are considered.

Out of the 24 STAs, 16 STAs have an uplink broadband traffic modeled as a File Transfer Protocol (FTP3) service 
[16] with a file size of $0.5 \mathrm{MB}$ and 8 STAs have low-latency traffic modeled as an augmented reality (AR) application [17], with a file size of 32 bytes and files arriving periodically at a frequency of $10 \mathrm{~ms}$. For simplicity, in the rest of the paper, the STAs with FTP3 traffic are referred to as broadband traffic stations, while the STAs with periodic file arrival traffic are referred to as low-latency traffic stations. There is a fundamental challenge when it comes to maintaining acceptable performance in this mixed traffic scenario: as STAs share the same channel, broadband stations generate most of the load in the system and might be scheduled for long transmissions while the low-latency stations, that should meet latency deadlines, might continuously find the channel busy.

In the baseline scenario, APs implement all the main IEEE 802.11ax features. When triggering uplink, APs spatially multiplex as many STAs as possible per transmission opportunity. For the PSR deployment, on top of the baseline functionalities, STAs can contend for channel access, but only when PSRbased channel access opportunities are identified. With the main objective of reducing the worst-case latencies of the low-latency traffic STAs, following the explanation introduced in the example given in Sec. [I] we assume that in the PSR scenario all APs enable PSR operations, and only low-latency traffic stations can grab those PSR opportunities.

Application files are generated by the traffic model and segmented into the transport layer, including the transport layer headers. The packet is then converted into internet protocol (IP) packets in the IP layer. The IP packets are then mapped into medium access control (MAC) layer frames and physical (PHY) layer symbols subsequently, with headers and overhead considered and variable PHY header sizes depending on transmission characteristics.

The channel model is based on the 3D Indoor Office ( $\mathrm{InH})$ channel model from the latest 3GPP Technical Report 38.901 [18], which accounts for 3D channel directionality in the large-scale and small-scale fading parameters. The algorithm used for MCS selection is a signal-to-interference-plus-noiseratio (SINR)-driven approach, in which we assume that the transmitting STA knows what the active and transmitting nodes are. All the APs employ a Round Robin scheduler, with strict priority given to low-latency STAs.

A summary of the system parameters is shown in Table \

\section{B. Deployment Density}

In this section, we consider three different deployment densities: higher, medium, and lower density, with $d$ in the scenario described in Fig. 2 varying from 10 to 20 and 30 meters, respectively, and traffic load of 100 Mbps for broadband stations.

Fig. 3 illustrates the impact of PSR on the worst file delays experienced by the STAs generating low-latency traffic. Overall, we can observe that, for all densities considered, when the 802.11 ax PSR feature is activated (solid curves), smaller delays can be achieved compared to baseline configurations without such feature. Indeed, when compared to the baseline 802.11ax system, the PSR feature is capable of reducing the 5\%-worst

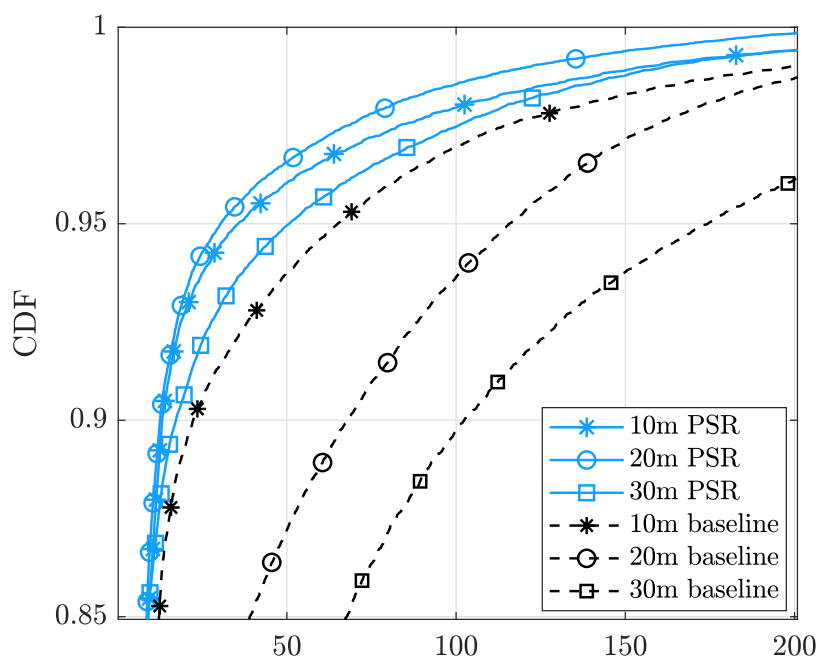

UL file delay (ms) for STAs with low-latency traffic.

Fig. 3. CDF of the uplink file delay for low-latency STAs, inter-AP distances $d=\{10,20,30\} \mathrm{m}$, and 100 Mbps broadband traffic load.

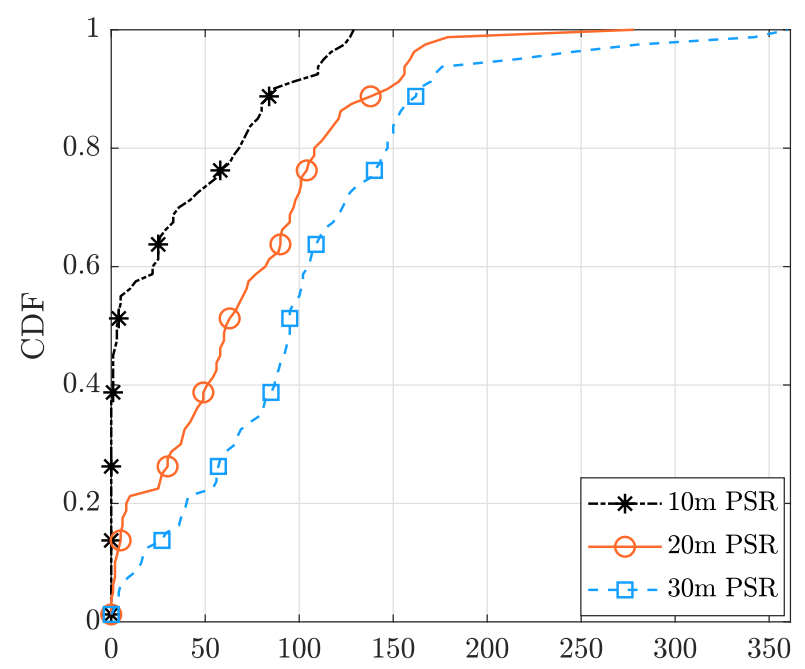

Num. of PSR TXOPs per STA with low-latency traffic.

Fig. 4. CDF of the number of PSR-based transmissions gained per low-latency STA, inter-AP distances $d=\{10,20,30\} \mathrm{m}$, and $100 \mathrm{Mbps}$ broadband traffic load.

delays by a factor of approximately $1.8 \times, 3.8 \times, 3.4 \times$ for interAP distances of 10, 20, and $30 \mathrm{~m}$, respectively.

The following conclusions can be made from Fig. 3 .

- For the baseline 802.11ax system, the worst-case delays are reduced as the deployment density increases. This is because the STAs are closer to their serving APs in denser scenarios, which allows the STAs to transmit using higher MCS levels and reduces their file transmission duration. In turn, this results in reduced channel access delays since the wireless channel becomes less occupied. Moreover, some devices might not be able to detect other ongoing transmissions in low-density scenarios due to the distance, which leads to a 


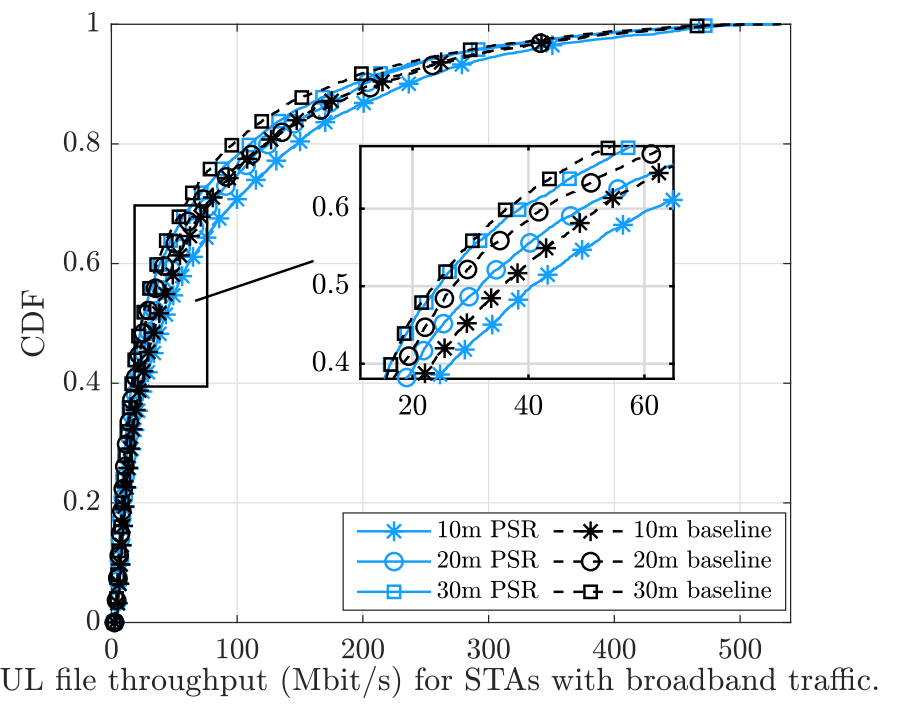

Fig. 5. CDF of the uplink file throughput for broadband STAs, inter-AP distances $d=\{10,20,30\} \mathrm{m}$, and $100 \mathrm{Mbps}$ broadband trafic load.

higher number of collisions and retransmissions [15].

- Instead, for the 802.11ax system with PSR capabilities, the worst-case file transmission delays are reduced when the inter-AP distance grows from $10 \mathrm{~m}$ to $20 \mathrm{~m}$. This behavior can be explained by observing Fig. 4, which illustrates the $\mathrm{CDF}$ of the number of PSR-based gained transmissions per station for each of the deployment densities of Fig. [3. Fig. 4 demonstrates that low-latency STAs have the lowest probability of gaining PSR-based transmissions in the densest deployment considered, with more than $40 \%$ of the stations not being capable of finding SR channel access opportunities. This happens because in the densest deployment scenario they i) are less likely to find SROssince the increased RPL means that they cannot satisfy the power constraint set by the AP granting the SR opportunity as per (3) - and/or ii) deem the medium as busy when-due to uplink transmissions from STAs triggered by the donor AP-their RPL is higher than the physical carrier sensing threshold.

- Furthermore, the results of Fig. 3 illustrate that the worstcase transmission delays under PSR increase when the interAP distances grow from $20 \mathrm{~m}$ to $30 \mathrm{~m}$. This is because the performance loss resulting from having higher distances between STAs and their serving APs-following the same arguments as the corresponding baseline scenario-outweighs the gains introduced by the slightly higher number of SROs found, as per Fig. 4 .

Fig. 5 shows the impact of PSR on the throughput of the broadband stations. Overall, it can be observed that broadband stations also benefit from the implementation of the PSR feature. This is a direct consequence of the reduced number of devices that contend simultaneously for the wireless medium, and the resultant reduced channel access delays. The rationale for this behavior is illustrated in Fig. 1 (b).

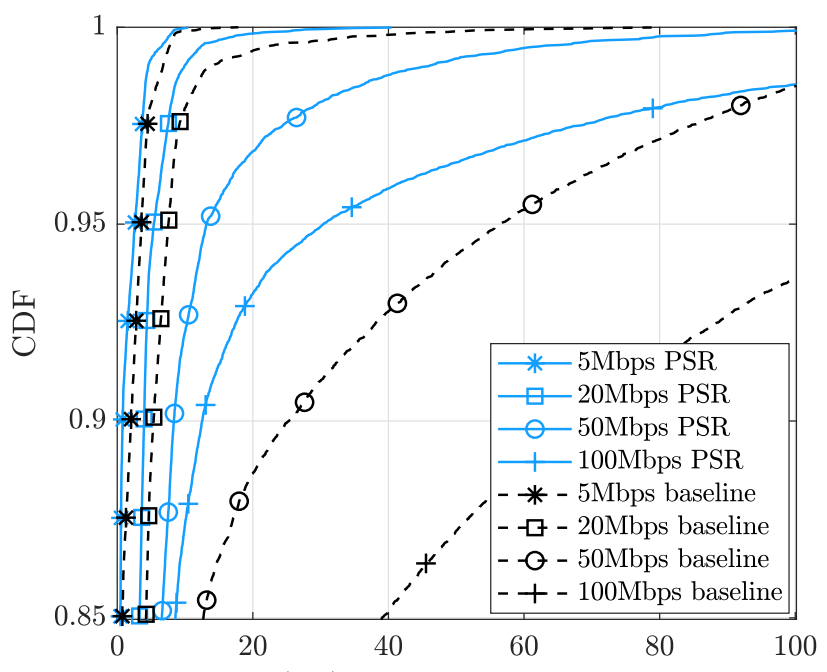

UL file delay (ms) for STAs with low-latency traffic.

Fig. 6. CDF of the uplink file delay for low-latency STAs, traffic loads of $\{5,20,50,100\} \mathrm{Mbps}$, and inter-AP distance of $20 \mathrm{~m}$.

\section{Traffic Load}

Fig. 6 quantifies the worst-case latency benefits of enabling PSR under different traffic loads. More concretely, Fig. 6 shows the CDF of the $15 \%$ worst-case uplink file delays for low-latency STAs when the application layer traffic offered for broadband stations varies from $5 \mathrm{Mbps}$ to $100 \mathrm{Mbps}$ in the scenario with $20 \mathrm{~m}$ inter-AP distances. When comparing the behavior of the systems with and without PSR capabilities, two distinct regimes can be observed in Fig. 6. First, for low and moderate traffic loads, i.e., up to $20 \mathrm{Mbps}$, both systems' latency performance is similar. This is because the wireless medium is generally available for transmission when devices transmit sporadically, which entails that devices do not need to contend for channel access for long periods. Second, for relatively high traffic loads, i.e., $50 \mathrm{Mbps}$ and above, the system with PSR capabilities provides substantial latency benefits. Specifically, the implementation of PSR allows reducing the $15 \%$ worst-case latencies by a factor of $\approx 4.6 \times$ when the offered traffic load reaches $100 \mathrm{Mbps}$. It is for these high traffic loads-where the wireless medium remains generally occupied - that allowing a more aggressive spatial reuse can provide remarkable latency benefits.

\section{Number of Antennas at the AP}

Fig. 7 shows the upper part of the CDF of the uplink file delay - which includes the median and worst-case latenciesfor the low-latency stations when equipping APs with 1, 2, 4 or 8 antennas. We consider a scenario with an inter-AP distance of $20 \mathrm{~m}$ and where the broadband stations offer a traffic load of $100 \mathrm{Mbps}$ to stress the system load. As one could expect, the file latencies of 802.11ax systems with and without PSR capabilities are generally reduced when the number of 


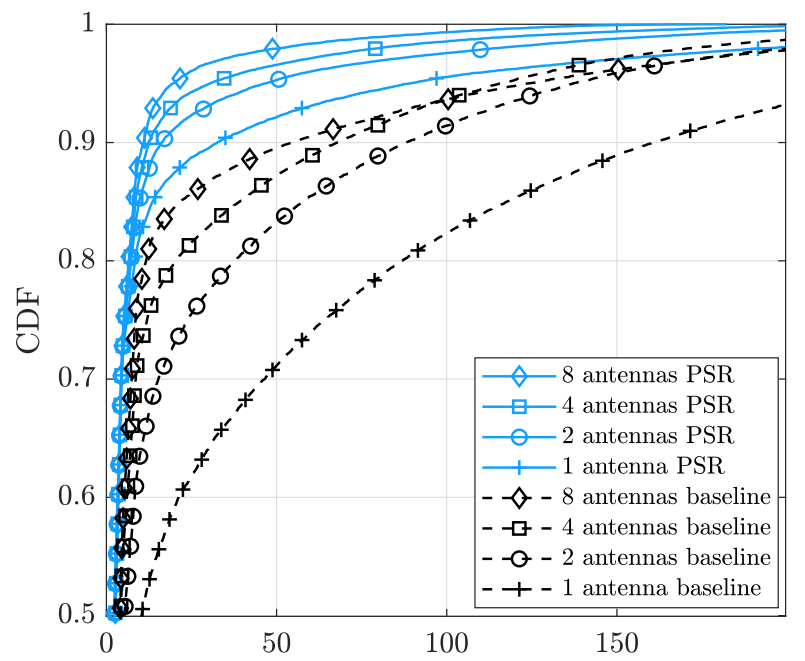

UL file delay (ms) for STAs with low-latency traffic.

Fig. 7. CDF of the uplink file delay for low-latency STAs, inter-AP distance $d=20 \mathrm{~m}$, traffic load of $100 \mathrm{Mbps}$, and various numbers of AP antennas.

antennas per AP grows We can also observe how, for a fixed number of AP antennas, a PSR-capable system always outperforms the corresponding baseline system.

Remarkably, the results of Fig. 7 demonstrate that for a large portion of the CDF- up to the 95th percentile-a PSRsystem with single-antenna APs can outperform a system without spatial reuse capabilities where the APs are equipped with eight antennas. In other words, allowing low-latency STAs to access the channel more aggressively is generally more effective than increasing the maximum AP throughput per transmission through multi-antenna capabilities for the scenarios under evaluation. Indeed, we can observe that the file latencies of PSR-capable systems are almost identical up to the 80th percentile of the CDF, regardless of the number of antennas per AP. Altogether, these results convey the important message that - for short-packet low-latency applications-the deployment of APs with PSR signal processing capabilities may be an appealing alternative to the deployment of the traditionally more costly multi-antenna APs.

\section{CONCLUSIONS}

In this paper, we have provided an extensive analysis of the benefits of enabling the PSR feature in 802.11ax WLANs in terms of reduced latency. Under realistic models, we evaluated the performance of PSR for different scenario densities, traffic loads, and AP antenna arrays. Our studies show that, for high-, medium-, and low-density deployments with high traffic loads, PSR can reduce worst-case latencies with respect to baseline 802.11ax operations by a factor $1.8,3.8$, and 3.4, respectively. The results also show that allowing a more aggressive spatial reuse in high-loaded scenarios provides the most remarkable

\footnotetext{
${ }^{2}$ The crossover between the four- and eight-antenna scenarios is due to the system scheduling as many STAs as possible. This is, in general, suboptimal, e.g., for APs with many antennas and spatial channel correlation among STAs.
}

latency benefits. Finally, we found that, for the scenarios under considerations, even single-antenna APs with PSR capabilities may outperform baseline 802.11ax multi-antenna APs, designating the PSR feature as an alternative to more costly multiantenna APs for low-latency applications. Possible future work includes investigating the performance of PSR when combined with novel IEEE 802.11 be features to further increase spatial reuse and reduce latency, such as coordinated beamforming and null steering [4], [5], [19].

\section{REFERENCES}

[1] (2020, Mar) Cisco Annual Internet Report (2018-2023) White Paper. [Online]. Available: https://www.cisco.com/c/en/us/solutions/collateral/executive-perspectives/annual-interne

[2] D.-J. Deng et al., "IEEE 802.11ax: highly efficient WLANs for intelligent information infrastructure," IEEE Communications Magazine, vol. 55 , no. 12 , pp. 52-59, 2017.

[3] "P802.11ax/D6.0 - IEEE Draft Standard for Information Technology - Telecommunications and Information Exchange Between Systems Local and Metropolitan Area Networks - Specific Requirements Part 11: Wireless LAN Medium Access Control (MAC) and Physical Layer (PHY) Specifications - Amendment 1: Enhancements for High Efficiency WLAN," IEEE 802.11, November 2019.

[4] A. Garcia-Rodriguez et al., "Performance of parameterized spatial reuse (PSR) with coordinated beamforming/null steering for $802.11 \mathrm{be}, "$ IEEE 802.11-20/0091r2, Jan. 2020.

[5] D. López-Pérez, A. Garcia-Rodriguez, L. Galati-Giordano, M. Kasslin, and K. Doppler, "IEEE 802.11be Extremely High Throughput: The Next Generation of Wi-Fi Technology Beyond 802.11ax," IEEE Communications Magazine, vol. 57, no. 9, pp. 113-119, 2019.

[6] E. Khorov, I. Levitsky, and I. F. Akyildiz, "Current status and directions of IEEE 802.11be, the future Wi-Fi 7," IEEE Access, vol. 8, pp. 88664 $88688,2020$.

[7] A. Garcia-Rodriguez, D. Lopez-Perez, L. Galati-Giordano, and G. Geraci, "IEEE 802.11be: Wi-Fi 7 Strikes Back," 2020.

[8] Q. Qu et al., "Survey and performance evaluation of the upcoming next generation WLANs standard - IEEE 802.11ax," Mobile Networks and Applications, vol. 24, no. 5, pp. 1461-1474, 2019.

[9] E. Khorov, A. Kiryanov, A. Lyakhov, and G. Bianchi, "A tutorial on IEEE 802.11ax high efficiency WLANs," IEEE Communications Surveys \& Tutorials, vol. 21, no. 1, pp. 197-216, 2018.

[10] M. S. Afaqui, E. Garcia-Villegas, and E. Lopez-Aguilera, "IEEE 802.11 ax: Challenges and requirements for future high efficiency WiFi," IEEE Wireless Communications, vol. 24, no. 3, pp. 130-137, 2016.

[11] F. Wilhelmi et al., "Spatial Reuse in IEEE 802.11ax WLANs," arXiv preprint arXiv:1907.04141, 2019.

[12] A. Valkanis, A. Iossifides, P. Chatzimisios, M. Angelopoulos, and V. Katos, "IEEE 802.11ax spatial reuse improvement: An interferencebased channel-access algorithm," IEEE Vehicular Technology Magazine, vol. 14, no. 2, pp. 78-84, 2019.

[13] Z. Shen et al., "Research and Performance Evaluation of Spatial Reuse Technology for Next Generation WLAN," in International Wireless Internet Conference. Springer, 2018, pp. 41-51.

[14] F. Wilhelmi, S. Barrachina-Muñoz, and B. Bellalta, "On the Performance of the Spatial Reuse Operation in IEEE 802.11ax WLANs," in 2019 IEEE Conference on Standards for Communications and Networking (CSCN). IEEE, 2019, pp. 1-6.

[15] E. Perahia and R. Stacey, Next generation wireless LANs: 802.11 n and 802.11ac. Cambridge university press, 2013.

[16] "Evolved Universal Terrestrial Radio Access (E-UTRA); Further advancements for E-UTRA physical layer aspects," $3 G P P$ TR 36.814, March 2017.

[17] "Study on physical layer enhancements for NR ultra-reliable and low latency case (URLLC)," 3GPP TR 38.824, March 2019.

[18] "Study on channel model for frequencies from 0.5 to $100 \mathrm{GHz}$," $3 G P P$ TR 38.901, June 2018.

[19] A. Garcia-Rodriguez et al., "Massive MIMO unlicensed: A new approach to dynamic spectrum access," IEEE Communications Magazine, vol. 56, no. 6, pp. 186-192, 2018. 